\title{
Introduction: Is an Interdisciplinary Field of Memory Studies Possible?
}

\author{
Adam D. Brown • Yifat Gutman • Lindsey Freeman • \\ Amy Sodaro • Alin Coman
}

Published online: 26 May 2009

(C) Springer Science + Business Media, LLC 2009

Keywords Memory $\cdot$ Memory studies $\cdot$ Interdisciplinary $\cdot$ Collective memory

Understanding the processes by which individuals and groups remember or forget the past has been a concern for centuries. However, over the past three decades, the study of memory has become an increasingly popular topic for scholarly inquiry. This surge in memory research has greatly contributed to the way in which we consider a broad range of issues from the most basic biological and cellular encoding and retrieval systems to the ways in which political and cultural systems facilitate the remembering or silencing of historical events. As a result, the concept of "memory" is now studied and taught across a wide range of academic disciplines from the natural and social sciences, the humanities, and the arts. In addition, the past three decades have witnessed an increase in the number of texts, scholarly journals, conferences, students, and practitioners interested in the understanding and function of memory. Of particular interest are burgeoning attempts among memory scholars seeking to collaborate across disciplinary lines. Scholarly organizations, special journal issues, edited books, professional meetings, and at least one academic journal look to promote interdisciplinary interactions. The identification and acknowledgment of the interdisciplinary interest in memory has been referred to as "memory studies."

Although the specific parameters or goals of memory studies remain somewhat unclear, there is a perception among many scholars that interactions across the traditional disciplinary boundaries have the potential to advance the study of memory by providing innovative ways

\footnotetext{
A. D. Brown $(\bowtie)$

Functional Neuroimaging Laboratory, Department of Psychiatry, Weill Medical College of Cornell University, 525 East 68th Street, Mailbox \# 140, New York, NY 10065, USA

e-mail: adb2004@med.cornell.edu

Y. Gutman • L. Freeman • A. Sodaro

Department of Sociology, The New School for School for Social Research, New York, NY, USA
}

A. Coman

Department of Psychology, The New School for School for Social Research, New York, NY, USA 
of looking at individual and social issues related to memory. Perhaps the clearest example is the creation of the peer-reviewed journal Memory Studies, which aims to cultivate dialog and comparison among scholars from a wide range of academic disciplines with the goal, in part, to identify areas that overlap or diverge (Hoskins et al. 2008).

For the established scholar, as well as the student of memory, the prospect of a field of memory studies holds great promise. It is difficult to imagine an area of one's life that is not shaped profoundly by memory, from our most personal and intimate recollections of the past, our sense of self and our ability to successfully execute daily routines, to the ways in which remembering communities construct the past through language, ceremonial and mundane practices, political policy, and physical structures. Yet, our respective disciplines are limited in addressing this complex nexus of questions due to methodological or theoretical constraints. Thus, the mobilization of memory studies, at first glance, has endless potential for interesting interactions and collaborations that reveal a larger piece of the puzzle.

We have witnessed how other interdisciplinary discussions on themes such as nationalism have greatly contributed to the advancement of knowledge. Like other fields of inquiry, such as gender studies and cultural studies, the field of memory studies has arisen from the political expediencies of the moment and the high political stakes of memory and the use of the past (Radstone 2008). Furthermore, the institutionalized study of transitional justice, conflict resolution, human rights, and memory studies comes as a response to the very real activities by various groups, governments, individuals, and other actors that are immediately and directly concerned with facing the past and its memory and incorporating that past - its victims, perpetrators, and physical remnants - into the present. The realm of memory "practice" is often very interdisciplinary: psychologists not only treat individuals traumatized by the past but also work with lawyers and social workers to create truth commissions and modes for taking testimony; policy makers are looking to history to understand the causes of past conflict and violence in order to help shape social and institutional structures that can prevent future conflicts; artists are working with human rights activists in the struggle to find ways to represent past trauma and atrocity; findings from scholarly research and activism are used by educators to shape the future. We in the academy can look to memory practitioners to discover how fruitful interdisciplinary collaborations are in practice.

While the promise of a field of memory studies is exciting, the efforts to mobilize a field in a coherent and productive manner provide a number of challenges. Conferences promise "interdisciplinary approaches" to a particular area within memory research, artists and neuroscientists find themselves sitting next to each other at a symposium, and students from a wide range of backgrounds attend presentations, but to what extent does the work of one discipline influence another's as a result of these meetings? Do these attempts at collaboration more often result in multidisciplinarity, rather than interdisciplinarity, in which scholars are exposed to other disciplines' perspectives, but little is transferred from one academic discipline to the next? Are the methods and assumptions found in one body of research unhelpful for scholars in other disciplines? Are the terms used in one discipline so different that students from one area of memory research require translators to help them decode the references and types of memory being discussed? Further, would scholars be able to agree on the basic definitions of a field, starting with the definition of memory?

The authors of this introduction represent a small group of memory researchers interested in whether there exists a field of memory studies and how interdisciplinarity in such a field might shape the academic institutional landscape. Having developed our own research interests and work in the cultural contexts and academic institutions at a time when the notion of memory studies is being considered, we began meeting to explore some of these issues and questions in greater detail. Our discussions led to the formation of a 
memory conference at the New School for Social Research, in February 2008, called "Is an Interdisciplinary Field of Memory Studies Possible?" which brought leading scholars of memory from a number of disciplines together to examine this question. The papers and discussions that came out of the conference continue to contribute to the ongoing debate over the state of memory studies today, as does this special journal issue.

\section{The Problem with Definitions}

As any student or scholar of memory knows, defining memory is particularly challenging. Even the most cursory survey of memory research reveals that the definition and function of memory is polymorphic both within and between academic disciplines, cultural institutions, and memory practitioners. The unit of analysis, methodology, and theoretical assumptions vary greatly. Although most psychologists agree that there are three types of long-term memory systems, the survey of memory research of Roediger and Wertsch (2008) reminds us that Tulving (2007) has cataloged 256 kinds of memory mentioned in psychology journals. The difficulty of defining memory becomes even thornier when seeking similarities between synaptic changes in Aplysia (Kandel 2007) and the memory located in museums and archives, studied by architects, sociologists, and historians. As one begins to seek a common set of definitions and taxonomy, one wonders if many of the academic disciplines are studying the same thing at all. A commonly agreed-upon definition remains problematic even when specific terms are shared between disciplines. Consider the study of collective memory. Since its emergence as a distinct field of study as a result of the pioneering work of Halbwachs (1980/1925), scholars in recent decades have demonstrated an enormous interest in collective memory. Both sociologists and psychologists have adopted the term and study it largely along disciplinary lines, with little agreement or sharing of findings between the two fields (Olick 1999; Hirst and Manier 2008; Hirst and Echterhoff 2008). Given the enormous range of memory interests and methodologies used to explore them, we suspect that "memory" as the object of a field of studies will not fit neatly into a single definition. Nor would it be productive to try to construct one. However, increased attempts between scholars and students of memory to establish where various understandings of memory overlap would be a fruitful step in facilitating the emergence of a language about memory that could be more readily shared among scholars.

\section{Is Memory Studies a Field?}

The question of whether or not memory studies is a field may be best answered by looking at the ways in which scholars and practitioners are using memory in current research that crosses the disciplines. Institutionally, a number of centers have emerged over the past few years that are especially interested in interdisciplinary approaches to memory. The Center for Interdisciplinary Memory research in Germany, directed by Harald Welzer, is comprised of an international group of scholars spanning the natural and social sciences, who are dedicated to collectively investigating issues related to autobiographical memory, trauma, and memorialization. Recently, the Warwick Center for Memory Studies was established under the direction of Andrew Hoskins, editor of Memory Studies, and is also comprised of international scholars from a wide range of disciplines. Washington University in St. Louis created the Luce Program in Individual and Collective Memory, offering students the opportunity to study issues related to personal and collective memory with faculty from 
psychology, philosophy, history, and English (for a full description see, Roediger and Wertsch 2008).

In some fields, interdisciplinary interactions are already well established. For example, almost every cognitive psychology text book will draw on findings from psychology, neuroscience, and behavioral physiology. More specifically, discoveries in medical and applied sciences have promoted ongoing collaborations between psychologists, computer scientists, neuroscientists, biologists, geneticists, and others. Not surprisingly, these partnerships have led to important advances in the assessment, treatment, and prevention of memory disorders. In the social sciences and humanities, media studies scholars look toward sociology as a way to conceptualize issues pertaining to memory and media. Sociologists in turn glean insights from media studies when thinking about issues of transmission, retention, and reformation of memories in the digital age. Sociologists also draw from philosophy, phenomenology, comparative literature, and architectural theory when studying spaces and practices of memory. However, the boundary between psychology and the natural sciences and sociology and the study of cultural practices is probably the most difficult to cross.

Some of the most interesting and useful interdisciplinary interactions can be observed among practitioners drawing on scholarly findings. Within the field of psychology, clinical psychologists have developed treatments that were originally conceptualized by cognitive scientists and physiologists. ${ }^{1}$ Legal scholars work with psychologists to develop better ways of understanding the fallibility of eyewitness testimony and confessions. Lawyers and judges work with biologists, neuroscientists, and psychologists to explore the role of neuroscience in the courtroom, with particular interest in memory and deception. Political scientists, legal scholars, and sociologists work with transitional justice groups to commemorate and recognize issues of human rights abuse. Oral historians and anthropologists work directly with individuals or groups to collect, archive, and examine narratives. Architects and city planners collaborate with municipalities in order to integrate the built environment with citizens' memory of place.

Taken together, the extant observations suggest that there are ongoing interdisciplinary interactions within a wide range of areas pertaining to memory, both on theoretical and applied levels. However, do these interactions and intersecting points of interest in memory call for a unified field? Or would it be best to keep things separate; to remain loosely organized, allowing for some kinds of memory research to overlap more than others? These were some of the questions that drove the design of our 2008 interdisciplinary conference, which brought together leading scholars of memory to consider the question of whether there is or can be an interdisciplinary field of memory studies and, if so, what it would look like.

\section{Is an Interdisciplinary Field of Memory Studies Possible?}

The 3-day conference brought together over 85 senior and junior scholars of memory from over 29 disciplines and 34 universities. In addition to keynote lectures by Dominick LaCapra and Richard McNally, the first day consisted of nine thematically organized panels, discussing important themes within memory studies such as: Silence, Media/Space, Identity, Power \& Truth, Trauma, and Theory. On the second day, three panels, The Individual, The Collective, and The In-Between, explored how memory occurs and is studied within the individual, the

\footnotetext{
${ }^{1}$ For example, the "gold standard" treatment for Posttraumatic Stress Disorder, a psychiatric disorder that is characterized by re-experiencing symptoms, is Prolonged Imaginal Exposure Therapy, in which clinicians systematically desensitize patients to their fear memories over the course of treatment.
} 
collective, and the realm in between. This was followed by a Synthesis panel composed of some of the leading scholars of memory studies in the country: Daniel Levy moderated; William Hirst, Jeffrey Olick, Robin Wagner-Pacifici, and Barbie Zelizer presented briefly on the conference and the state of memory studies within their own disciplines; and Ann Louise Shapiro and Eviatar Zerubavel responded. These scholars were asked to reflect on the conference and the broader question of whether an interdisciplinary field of memory studies is possible. In this final session, scholars offered a wide variety of responses from enthusiastic support to trepidation and rejection. Concerns were raised about the various objects of study, methods and goals of research, and analyses that exist in divergent disciplines.

\section{Should Memory Studies be a Field? Responses}

Whether the ongoing interdisciplinary work described above and attempted by this conference necessitates a unified field remains open to debate. A number of advantages associated with establishing a field of memory studies was raised by the synthesis panel. The first is the ability for scholars and practitioners across disciplines to more readily share findings and analyses in a more logical and accessible way. Both Hirst and Zerubavel, for example, agreed that their work had benefitted from looking to other disciplines. For Hirst, this means a more directly interdisciplinary approach, where psychology actively contributes to collective memory studies in terms of reception and identity; for Zerubavel, this means a more multidisciplinary approach that uses work and theoretical assumptions from other disciplines, while maintaining disciplinary boundaries. Ideally this sort of inter- or multidisciplinary consideration might lead to a more comprehensive and nuanced understanding of certain memory issues, such as reception. However, a number of disadvantages were raised as well. Although scholars and students from a wide range of disciplines study memory, they have difficulty agreeing on the very basic definitions and assumptions, as we saw in several of the interdisciplinary panels and as was underlined by members of the synthesis panel. There are clearly some disciplines that overlap more easily with others, but collaborations between sociology and psychology are more difficult and attempting to find a common set of assumptions and methods might be unproductive. Wagner-Pacifici brought up the important differences to consider regarding the distance each discipline has to the object of study, and how these diverging proximities may make a coherent field difficult. At the same time, these differences also increase scholars' self-awareness of the limitations of their respective disciplinary lens and assume a bigger picture which involves various levels and scales of inquiry.

There may also be trepidation between the disciplines about forming a field of memory studies, when researchers from different disciplinary backgrounds are interested in divergent aspects of memory. For example, psychologists have little interest in how cognitive processes and representations in the mind relate to conceptualizations of memory in buildings and artifacts. And, sociologists are not compelled to study neurological pathways that inhibit remembering. But there are also more pressing political concerns about forming a distinct and separate field, as Zelizer argued. Scholars might not want to shed the prestige and legitimacy that they are granted from more established disciplines. They also might want to avoid being pigeonholed into a specific field of study, such as memory, preferring a wider range of inquiry, such as history, political science, or literature. There are questions of disciplinary authority, university placements, publications, and funding to consider. Speaking to these worries, Olick discussed a new "canon" of collective memory studies that he is currently editing that has raised a good deal of skepticism and uneasiness, as it will ultimately determine the founding texts of the field. 
Another fear is that a field of memory studies would not be productive but merely a passing trend or an institutional construction that creates diligence and nothing more.

\section{Conclusion}

The many divergent opinions that were expressed during the synthesis panel revealed a paradox among those who study and practice memory. On the one hand, interactions are frequently taking place between disciplines and between scholars and practitioners. These interactions may be the result, or the cause, of increased interest in creating a field of memory studies. However, as an interest in memory studies grows institutionally, there are many competing ideas of how, and if, those who study memory should seek to formally establish a unified field. Rather than conclude that there is a single way for memory researchers to proceed, we imagine several directions this growing field of memory will take.

One such direction is to establish a common set of definitions and taxonomy for creating a shared vocabulary. When the authors began meeting, they experienced great difficulty discussing memory across disciplines. This was particularly true for those terms that are shared, but have different meanings. ${ }^{2}$ However, as the meetings continued and the authors gained an understanding of each discipline's distinct definitions, they began to recognize similarities and possible contributions of one discipline to the others. In this issue, Coman, Brown, Koppel, and Hirst (2009) consider how psychology might complement the discussion of collective memory within the other social sciences in "Collective Memory from a Psychological Perspective."

A field of memory studies would also benefit from sharing methodologies and techniques for investigating memory. As previously mentioned, some disciplines overlap more easily than others, but this is not to say that innovative ways of sharing methodologies could not lead to novel ways of approaching problems. As with methodology, developing spheres of overlapping theoretical interest would be central to defining areas of interdisciplinary work among memory scholars.

For example, forgetting is a topic within memory studies that might benefit from shared theoretical assumptions. In this issue, both Goldfarb (2009), "Resistance and Creativity in Social Interaction: For and Against Memory in Poland, Israel-Palestine and the United States," and Poole (2009), "Enacting Oblivion," are concerned with the political aspects of forgetting. Poole illustrates how political projects intend to draw a thick black line between present and past, while Goldfarb illustrates the phenomena of individual or collective forgetting as a result of political amnesia, which is sometimes necessary for social change and creative political action. Here the work of philosophy and sociology contribute to our understanding of history, political science and law. The article by Susan Pearce follows the guidance of Jeffrey Olick, among others, who believe that not enough of the writings on memory incorporate ethnographic approaches, especially on the occasions of significant anniversary commemorations. Her study of "The Polish Solidarity Movement in Retrospect: In Search of a Mnemonic Mirror" contributes to this lacuna.

Another option is not to require a commitment to a systematically coherent inquiry, but rather to an interdisciplinary focus; this is the approach taken by the authors of the journal Memory Studies, as well as the new memory studies programs that have developed.

\footnotetext{
${ }^{2}$ Such as the way in which psychologists and sociologists refer to collective memory (Olick 1999; Hirst and Manier 2008)
} 
Among the papers in this issue, certain themes arise across disciplines and the treatment of these themes can contribute to a deeper understanding of memory and could potentially lead to cross-disciplinary research. For example, Kirkbride's (2009) paper "Chainmaking: A Note on Ornament, Intelligence and Building" and its companion piece by Kirkbride and Mattern (2009), "Chainbuilding: A New Building for the New New School," address technologies of memory, specifically architecture, as an organizing mechanism for both individual and collective memories. Similarly, Landsberg (2009) in "Memory, Empathy and the Politics of Identification," and Kang (2009) in "The Ur-History of Media Space: Walter Benjamin and the Information Industry in Nineteenth-Century Paris" are concerned with the way new media technologies, such as film and the commercial press, have enabled new modes of formulating memory that have the potential to alter what we as individuals "remember" and can change our group and individual identifications. The concept of technologies of memory may enable fruitful modes of speaking across disciplinary boundaries. As these papers argue, architectural "technologies" are used by both the individual and the institution just as mass media technologies fundamentally rupture what was once a close relationship between group identification and individual memories. In both cases, the technologies of memory are key to understanding the individual's and collective's relationship to the past.

Another way to benefit from a conversation beyond disciplines is to explore both disciplinary work and "the in-between"-the boundary between one disciplinary inquiry and another. This idea was expressed from different perspectives both by Olick and Zerubavel during the conference.

Perhaps the least demanding model for an interdisciplinary collaboration, which still yields significant benefit to the study of memory, is to place disciplinary inquiry within an interdisciplinary context. This approach advocates a certain self-reflectivity regarding the lens of examination, taking into consideration the assumptions and methods that each discipline enforces and how these might reveal or obstruct significant knowledge. This option, which might be referred to as a multidisciplinary focus rather than an inter- or transdisciplinary one, was suggested at the conference by Wagner-Pacifici and Shapiro.

On the arch that stretches between the interdisciplinary model that emphasizes commonalities and interactions to the proposal that focuses on differences, there are many opportunities for memory scholars, and especially for students entering the "field," to better comprehend, advance, and shape the study of memory.

\section{References}

Coman, A., Brown, A. D., Koppel, J., \& Hirst, W. (2009). Collective memory from a psychological perspective. International Journal of Politics, Culture, and Society, 22(2). doi:10.1007/s10767-0099057-9.

Goldfarb, J. C. (2009). Resistance and creativity in social interaction: For and against memory in Poland, Israel-Palestine and the United States. International Journal of Politics, Culture, and Society, 22(2).

Halbwachs, M. (1980). Collective memory. New York: Harper \& Row. doi:10.1007/s10767-009-9055-y.

Hirst, W., \& Echterhoff, G. (2008). Creating shared memories in conversation: toward a psychology of collective memory. Social Research, 75, 183-216.

Hirst, W., \& Manier, D. (2008). Towards a psychology of collective memory. Memory, 16, 183-200.

Hoskins, A., Barnier, A., Kansteiner, W., \& Sutton, J. (2008). Editorial. Memory Studies, 1, 5-7.

Kandel, E. (2007). In Search of Memory: The Emergence of a New Science of Mind. New York: W.W. Norton \& Company.

Kang, J. (2009). The Ur-history of media space: Walter Benjamin and the information industry in nineteenthcentury Paris. International Journal of Politics, Culture, and Society, 22(2). doi:10.1007/s10767-0099052-1. 
Kirkbride, R. (2009). Chainmaking: A note on ornament, intelligence and building. International Journal of Politics, Culture, and Society, 22(2). doi:10.1007/s10767-009-9053-0.

Kirkbride, R., \& Mattern, S. (2009). Chainbuilding: A new building for the new New School. International Journal of Politics, Culture, and Society, 22(2). doi:10.1007/s10767-009-9054-z.

Landsberg, A. (2009). Memory, empathy and the politics of identification. International Journal of Politics, Culture, and Society, 22(2). doi:10.1007/s10767-009-9056-x.

Olick, J. (1999). Collective memory: the two cultures. Sociological Theory, 7, 333-348.

Poole, R. (2009). Enacting oblivion. International Journal of Politics, Culture, and Society, 22(2). doi:10.1007/s10767-009-9058-8.

Radstone, S. (2008). Memory studies: For and against. Memory Studies, 1, 31-39.

Roediger, H. W., III, \& Wertsch, J. (2008). Creating a new discipline of memory studies. Memory Studies, 1, 9-22.

Tulving, E. (2007). Are there 256 kinds of memory? In J. S. Nairne (Ed.), The foundations of remembering: essays in honor of Henry L. Roediger, III (pp. 39-52). New York: Psychology Press. 